American Journal of Applied Sciences 4 (6): 371-373, 2007

ISSN 1546-9239

(C) 2007 Science Publications

\title{
A Fixed Point Theorem for Contraction Type Mappings in Menger Spaces
}

\author{
Servet Kutukcu \\ Department of Mathematics, Faculty of Science and Arts, Ondokuz Mayis University \\ Kurupelit, 55139 Samsun, Turkey
}

\begin{abstract}
We proved a common fixed point theorem for a sequence of self maps satisfying a new contraction type condition in Menger spaces, results extended and generalize some known results in metric spaces and fuzzy metric spaces.
\end{abstract}

Key words: Fixed point, contraction map, Menger probabilistic metric space

\section{INTRODUCTION}

There have been a number of generalizations of metric space. One such generalization is Menger space introduced in 1942 by Menger ${ }^{[1]}$ who was use distribution functions instead of nonnegative real numbers as values of the metric. Schweizer and Sklar ${ }^{[2]}$ studied this concept and gave some fundamental results on this space. The important development of fixed-point theory in Menger spaces was due to Sehgal and Bharucha-Reid ${ }^{[3]}$. The study of common fixed points of maps satisfying some contractive type condition has been at the centre of vigorous research activity. It is observed by many authors ${ }^{[3,4-10]}$ that contraction condition in metric space may be translated into probabilistic metric space endoved with min norms. The purpose of this was to define and investigate a new class of self-maps satisfying a new contraction type condition in Menger spaces.

Preliminaries: We recall some definitions and known results in Menger probabilistic metric space. For more details, we refer the readers to ${ }^{[1,4-9,11,12]}$.

Definition 1: A triangular norm * (shorty t-norm) is a binary operation on the unit interval $[0,1]$ such that for all $a, b, c, d \in[0,1]$ the following conditions are satisfied:

(a) $a * 1=a$,

(b) $a * b=b * a$,

(c) $a * b \leq c * d$ whenever $a \leq c$ and $b \leq d$,

(d) $\mathrm{a} *(\mathrm{~b} * \mathrm{c})=(\mathrm{a} * \mathrm{~b}) * \mathrm{c}$.

Some examples of t-norms are $a * b=\max \{a+b-1,0\}$ and $a * b=\min \{a, b\}$.
Definition 2: A distribution function is a function $F:[-\infty, \infty] \rightarrow[0,1]$ which is left continuous on $\mathfrak{R}$, non-decreasing and $F(-\infty)=0, F(\infty)=1$. If $X$ is a nonempty set, $F: X \times X \rightarrow \Delta$ is called a probabilistic distance on $X$ and $F(x, y)$ is usually detoned by $F_{x y}$.

Definition $3\left({ }^{[1]}\right)$ : (see also[1-3,9]) The ordered pair $(X, F)$ is called a probabilistic semimetric space (shortly PSM-space) if $X$ is a nonempty set and $F$ is a probabilistic distance satisfying the following conditions: for all $x, y, z \in X$ and $t, s>0$,

(PM-1) $F_{x y}(t)=H(t) \Leftrightarrow x=y$,

(PM-2) $F_{x y}=F_{y x}$.

If, in addition, the following inequality takes place:

$(\mathrm{PM}-3) F_{x z}(t)=1, F_{z y}(s)=1 \Rightarrow F_{x y}(t+s)=1$, then $(X, F)$ is called a probabilistic metric space.

The ordered triple $(X, F, *)$ is called Menger probabilistic metric space (shortly Menger space) if $(X, F)$ is a PM-space, $*$ is a t-norm and the following condition is also satisfies: for all $x, y, z \in X$ and $t, s>0$, (PM-4) $F_{x y}(t+s) \geq F_{x z}(t) * F_{z y}(s)$. For every PSM-space $(X, F)$, we can consider the sets of the form $U_{\varepsilon, \lambda}=\{(x, y)$ $\left.\in X \times X: F_{x y}(\varepsilon)>1-\lambda\right\}$.

The family $\left\{U_{\varepsilon, \lambda}\right\}_{\varepsilon>0, \lambda \in(0,1)}$ generates a semi uniformity denoted by $U_{F}$ and a topology $\tau_{F}$ called the F-topology or the strong topology. Namely, $A \in \tau_{F}$ iff $\forall x \in A \exists \varepsilon>0$ and $\lambda \in(0,1)$ such that $U_{\varepsilon, \lambda}(\mathrm{x}) \subset A$. $U_{F}$ is also generated by the family $\left\{V_{\delta}\right\}_{\delta>0}$ where $V_{\delta}:=U_{\delta, \delta}$ $\left({ }^{[2]}\right)$.

In ${ }^{[13]}$, it is proved if $\sup _{t<0}(\mathrm{t} * \mathrm{t})=1$, then $U_{F}$ is a uniformity, called F-uniformity, which is metrizable. The F-topology is generated by the F-uniformity and is determined by the F-convergence: $x_{n} \rightarrow x$

Corresponding Author: Servet Kutukcu, Department of Mathematics, Faculty of Science and Arts, Ondokuz Mayis University, Kurupelit, 55139 Samsun, Turkey 
$\Leftrightarrow F_{x_{n} x}(t) \rightarrow 1, \forall t>0$.

Definition $4\left({ }^{[2]}\right)$ : A sequence $\left\{x_{n}\right\}$ in a Menger space $(X, F, *)$ is called converge to a point $x$ in $X$ (written as $\left.x_{n} \rightarrow x\right)$ if for every $\varepsilon>0$ and $\lambda \in(0,1)$, there is an integer $n_{0}=n_{0}(\varepsilon, \lambda)$ such that $F_{x_{n} x}(\varepsilon)>1-\lambda$ for all $n \geq$ $n_{0}$. The sequence called Cauchy if for every $\varepsilon>0$ and $\lambda$ $\in(0,1)$, there is an integer $n_{0}=n_{0}(\varepsilon, \lambda)$ such that $F_{x_{n} x_{m}}(\varepsilon)>1-\lambda$ for all $n, m \geq n_{0}$. A Menger space $(X, F, *)$ is said to be complete if every Cauchy sequence in it converges to a point of it.

Lemma $1\left({ }^{[9]}\right)$ : Let $\left\{x_{n}\right\}$ be a sequence in a Menger space $(X, F, *)$ with continuous t- norm $*$ and $t * t \geq t$. If there exists a constant $\alpha \in(0,1)$ such that $F_{x_{n} x_{n+1}}(\alpha t) \geq F_{x_{n-1} x_{n}}(t)$ for all $t>0$ and $n=1,2 \ldots$, then $\left\{x_{n}\right\}$ is a Cauchy sequence in $X$.

Lemma $2\left({ }^{[9]}\right)$ : Let $(X, F, *)$ be a Menger space. If there exists a constant $\alpha \in(0,1)$ such that $F_{x y}(\alpha t) \geq F_{x y}(t)$ for all $x, y \in X$ and $t>0$, then $x=y$.

Remark 1: In a Menger space $(X, F, *)$, if $t * t \geq t$ for all $t \in[0,1]$ then $a * b=\min \{a, b\}$ for all $a, b \in[0,1]$ and it is well known that such t-norm is continuous.

\section{RESULTS}

Theorem 1: Let $\left\{T_{n}\right\}, \mathrm{n}=1,2, \ldots$ be a sequence of mappings of a complete Menger space $(X, F, *)$ into itself with $t * t \geq t$ for all $t \in[0,1]$ and $S: X \rightarrow X$ be a continuous mapping such that $T_{n}(X) \subseteq S(X)$ and $S$ is commuting with each $T_{n}$. If there exists a constant $\alpha \in$ $(0,1)$ such that for any two mappings $T_{i}$ and $T_{j}$ $\min \left\{F_{T_{i} x T_{j} y}^{2}(\alpha t), F_{S x T_{i} x}(\alpha t) F_{S y T_{j} y}(\alpha t), F_{S y T_{j} y}^{2}(\alpha t)\right\}+a$ $F_{S y T_{j} y}(\alpha t) \quad F_{S x T_{j} y}(2 \alpha t) \geq\left[\begin{array}{lll}p & F_{S x T_{i} x}(t)+q & F_{S x S y}(t)\end{array}\right]$ $F_{S x T_{j} y}(2 \alpha t)$

holds for all $x, y \in X$ and $0<p, q<1$ and $0 \leq \mathrm{a}<1$ such that $p+q-a=1$, then there exists a unique common fixed point for all $T_{n}$ and $S$.

Proof: Let $x_{0}$ be an arbitrary point of $X$ and $\left\{x_{n}\right\}$ be a sequence defined by $S x_{n}=T_{n} x_{n-1}, n=1,2, \ldots$ Then for each $t>0$ and $0<\alpha<1$, we have

$$
\begin{gathered}
\min \left\{F_{T_{1} x_{0} T_{2} x_{1}}^{2}(\alpha t), F_{S x_{0} T_{1} x_{0}}(\alpha t) F_{S x_{1} T_{2} x_{1}}(\alpha t), F_{S x_{1} T_{2} x_{1}}^{2}(\alpha t)\right\}+ \\
a F_{S x_{1} T_{2} x_{1}}(\alpha t) F_{S x_{0} T_{2} x_{1}}(2 \alpha t) \geq
\end{gathered}
$$

$$
\begin{gathered}
{\left[p F_{S x_{0} T_{1} x_{0}}(t)+q F_{S x_{0} S x_{l}}(t)\right] F_{S x_{0} T_{2} x_{1}}(2 \alpha t) \text { and }} \\
\min \left\{F_{S x_{1} S x_{2}}^{2}(\alpha t), F_{S x_{0} S x_{1}}(\alpha t) F_{S x_{1} S x_{2}}(\alpha t), F_{S x_{l} S x_{2}}^{2}(\alpha t)\right\}+ \\
a F_{S x_{1} S x_{2}}(\alpha t) F_{S x_{0} S x_{2}}(2 \alpha t) \geq\left[p F_{S x_{0} S x_{1}}(t)+\right. \\
\left.q F_{S x_{0} S x_{1}}(t)\right] F_{S x_{0} S x_{2}}(2 \alpha t) .
\end{gathered}
$$

Thus, it follows that

$$
\begin{gathered}
\min \left\{F_{S x_{1} S x_{2}}^{2}(\alpha t), F_{S x_{0} S x_{1}}(\alpha t) F_{S x_{1} S x_{2}}(\alpha t)\right\}+a F_{S x_{1} S x_{2}}(\alpha t) \\
F_{S x_{0} S x_{2}}(2 \alpha t) \geq(p+q) F_{S x_{0} S x_{1}}(t) F_{S x_{0} S x_{2}}(2 \alpha t) \text { and } \\
F_{S x_{1} S x_{2}}(\alpha t) \min \left\{F_{S x_{1} S x_{2}}(\alpha t), F_{S x_{0} S x_{1}}(\alpha t)\right\}+a F_{S x_{1} S x_{2}}(\alpha t) \\
F_{S x_{0} S x_{2}}(2 \alpha t) \geq(p+q) F_{S x_{0} S x_{1}}(t) F_{S x_{0} S x_{2}}(2 \alpha t) .
\end{gathered}
$$

Since $F_{S x_{0} S x_{2}}(2 \alpha t) \geq \min \left\{F_{S x_{0} S x_{1}}(\alpha t), F_{S x_{l} S x_{2}}(\alpha t)\right\}$, we have

$$
\begin{gathered}
F_{S x_{1} S x_{2}}(\alpha t) F_{S x_{0} S x_{2}}(2 \alpha t)+a F_{S x_{1} S x_{2}}(\alpha t) F_{S x_{0} S x_{2}}(2 \alpha t) \geq \\
(p+q) F_{S x_{0} S x_{1}}(t) F_{S x_{0} S x_{2}}(2 \alpha t) \text { and } \\
(1+a) F_{S x_{1} S x_{2}}(\alpha t) F_{S x_{0} S x_{2}}(2 \alpha t) \\
\geq(p+q) F_{S x_{0} S x_{1}}(t) F_{S x_{0} S x_{2}}(2 \alpha t) .
\end{gathered}
$$

Since $p+q-a=1$, we have $F_{S x_{1} S x_{2}}(\alpha t) \geq F_{S x_{0} S x_{1}}(t)$.

By induction, $F_{S x_{n} S x_{n+1}}(\alpha t) \geq F_{S x_{n-1} S x_{n}}(t), n=1,2, \ldots$ Thus, by Lemma $1,\left\{S x_{n}\right\}$ is a Cauchy sequence in $X$. Since $X$ is complete, there exists some $u \in X$ such that $S x_{n} \rightarrow u$. Since $S x_{n}=T_{n} x_{n-1},\left\{T_{n} x_{n-1}\right\}$ also converges to $u$. Since $S$ commutes with each $T_{n}$, using (3.1), we have

$$
\begin{gathered}
\min \left\{F_{S S x_{n} T_{k} u}^{2}(\alpha t), F_{S S x_{n-1} S S x_{n}}(\alpha t) F_{S u T_{k} u}(\alpha t), F_{S u T_{k} u}^{2}(\alpha t)\right\}+ \\
a F_{S u T_{k} u}(\alpha t) F_{S S x_{n-1} T_{k} u}(2 \alpha t) \geq \\
{\left[p F_{S S x_{n-1} S S x_{n}}(t)+q F_{S S x_{n-1} S u}(t)\right] F_{S S x_{n-1} T_{k} u}(2 \alpha t) .}
\end{gathered}
$$

Using the continuity of $S$ and taking limits on both sides, we have

$$
\begin{gathered}
\min \left\{F_{S u T_{k} u}^{2}(\alpha t), F_{S u S u}(\alpha t) F_{S u T_{k} u}(\alpha t), F_{S u T_{k} u}^{2}(\alpha t)\right\}+ \\
a F_{S u T_{k} u}(\alpha t) F_{S u T_{k} u}(2 \alpha t) \geq \\
{\left[p F_{S u S u}(t)+q F_{S u S u}(t)\right] F_{S u T_{k} u}(2 \alpha t) \text { and so } F_{S u T_{k} u}^{2}(\alpha t)+} \\
a F_{S u T_{k} u}(\alpha t) F_{S u T_{k} u}(2 \alpha t) \geq(p+q) F_{S u T_{k} u}(2 \alpha t) .
\end{gathered}
$$

Since $F_{S u T_{k} u}(2 \alpha t) \geq \min \left\{F_{S u S u}(\alpha t), F_{S u T_{k} u}(\alpha t)\right\}=$ $F_{S u T_{k} u}(\alpha t)$, we have

$$
\begin{gathered}
(1+a) F_{S u T_{k} u}^{2}(2 \alpha t)=F_{S u T_{k} u}^{2}(2 \alpha t)+a F_{S u T_{k} u}(2 \alpha t) \\
F_{S u T_{k} u}(2 \alpha t) \geq(p+q) F_{S u T_{k} u}(2 \alpha t)
\end{gathered}
$$

and hence $F_{S u T_{k} u}(2 \alpha t) \geq 1$ for all $\alpha \in(0,1)$ and $t>0$. Therefore $S u=T_{k} u$ for any fixed integer $k$. Moreover, $\min \left\{F_{S x_{n} T_{k} u}^{2}(\alpha t), F_{S x_{n-1} S x_{n}}(\alpha t) F_{S u T_{k} u}(\alpha t), F_{S u T_{k} u}^{2}(\alpha t)\right\}+$ 


$$
\begin{gathered}
a F_{S u T_{k} u}(\alpha t) F_{S x_{n-1} T_{k} u}(2 \alpha t) \geq \\
{\left[p F_{S x_{n-1} S x_{n}}(t)+q F_{S x_{n-1} S u}(t)\right] F_{S x_{n-1} T_{k} u}(2 \alpha t) .}
\end{gathered}
$$

Taking the limits on both sides, we have

$$
\begin{gathered}
\min \left\{F_{u T_{k} u}^{2}(\alpha t), F_{u u}(\alpha t) F_{S u S u}(\alpha t), F_{S u S u}^{2}(\alpha t)\right\}+a F_{S u S u}(\alpha t) \\
F_{u T_{k} u}(2 \alpha t) \geq\left[p F_{u u}(t)+q F_{u S u}(t)\right] F_{u T_{k} u}(2 \alpha t)
\end{gathered}
$$

and so

$$
F_{u T_{k} u}^{2}(\alpha t)+a F_{u T_{k} u}(2 \alpha t) \geq\left[p+q F_{u S u}(t)\right] F_{u T_{k} u}(2 \alpha t) .
$$

Thus, it follows that $F_{u T_{k} u}(2 \alpha t) \geq 1$ for all $\alpha \in(0,1)$ and $t>0$. Therefore $u=S u=T_{k} u$ for any fixed integer $k$. Thus $u$ is a common fixed point of $S$ and $T_{n}$ for $n=$ $1,2, \ldots$

For uniquenesses, let $v$ be another common fixed point of $S$ and $T_{n}$ for $n=1,2, \ldots$ Using (3.1), we have

$$
\begin{gathered}
\min \left\{F_{u v}^{2}(\alpha t), F_{S u u}(\alpha t) F_{S v v}(\alpha t), F_{S v v}^{2}(\alpha t)\right\}+ \\
a F_{S v v}(\alpha t) F_{S u v}(2 \alpha t) \geq \\
{\left[p F_{S u u}(t)+q F_{S u S v}(t)\right] F_{S u v}(2 \alpha t) \text { and }} \\
F_{u v}^{2}(\alpha t)+a F_{u v}(2 \alpha t) \geq\left[p+q F_{u v}(t)\right] F_{u v}(2 \alpha t) .
\end{gathered}
$$

So $F_{u v}(2 \alpha t) \geq 1$ for all $\alpha \in(0,1)$ and $t>0$. Hence, by Lemma $2, u=v$. This completes the proof. If we take $a$ $=0$ in the main Theorem, we have the following:

Corollary 1: Let $\left\{T_{n}\right\}, \mathrm{n}=1,2, \ldots$ be a sequence of mappings of a complete Menger space $(X, F, *)$ into itself with $t * t \geq t$ for all $t \in[0,1]$ and $S: X \rightarrow X$ be a continuous mapping such that $T_{n}(X) \subseteq S(X)$ and $S$ is commuting with each $T_{n}$. If there exists a constant $\alpha \in$ $(0,1)$ such that for any two mappings $T_{i}$ and $T_{j}$

$$
\begin{gathered}
\min \left\{F_{T_{i} x T_{j} y}^{2}(\alpha t), F_{S x T_{i} x}(\alpha t) F_{S y T_{j} y}(\alpha t), F_{S y T_{j} y}^{2}(\alpha t)\right\} \geq \\
{\left[p F_{S x T_{i} x}(t)+q F_{S x S y}(t)\right] F_{S x T_{j} y}(2 \alpha t)}
\end{gathered}
$$

holds for all $x, y \in X$ and $0<p, q<1$ such that $p+q=1$, then there exists a unique common fixed point for all $T_{n}$ and $S$.

Proof: It is easy to verify from Theorem 1 . If we take $a$ $=0$ and $S=I_{X}$ (the identity map on $X$ ) in the main Theorem, we have the following:

Corollary 2: Let $\left\{T_{n}\right\}, \mathrm{n}=1,2, \ldots$ be a sequence of mappings of a complete Menger space $(X, F, *)$ into itself with $t * t \geq t$ for all $t \in[0,1]$. If there exists a constant $\alpha \in(0,1)$ such that for any two mappings $T_{i}$ and $T_{j}$

$$
\begin{gathered}
\min \left\{F_{T_{i} x T_{j} y}^{2}(\alpha t), F_{x T_{i} x}(\alpha t) F_{y T_{j} y}(\alpha t), F_{y T_{j} y}^{2}(\alpha t)\right\} \geq \\
{\left[p F_{x T_{i} x}(t)+q F_{x y}(t)\right] F_{x T_{j} y}(2 \alpha t)}
\end{gathered}
$$

holds for all $x, y \in X$ and $0<p, q<1$ such that $p+q=1$, then for any $x_{0} \in X$ the sequence $\left\{x_{n}\right\}=\left\{T_{n} x_{n-1}\right\}, n=1$, $2, \ldots$ converges and its limit is the unique common fixed for all $T_{n}$.

Proof: Existance and uniquess of common fixed point follows from Theorem 1. Convergence of the sequence $\left\{x_{n}\right\}$ can be proved as in Theorem 1 .

\section{REFERENCES}

1. Menger, K., 1942. Statistical metric. Proc. Nat. Acad. Sci., 28: 535-537.

2. Schweizer, B. and A. Sklar, 1960. Statistical metric spaces. Pasific J. Math., 10: 313-334.

3. Sehgal, V. and A. Bharucha-Reid, 1972. Fixed points of contraction mappings on probabilistic metric spaces. Math. System Theory, 6: 97-102.

4. Hadzic, O., 1995. Fixed point theory in probabilistic metric spaces, Serbian Academy of Science and Arts, Novi Sad University.

5. Hadzic, O. and E. Pap, 2001. Fixed point theory in probabilistic metric spaces, Kluwer Acad. Publ., Dordrecht.

6. Kutukcu, S., 2006. A fixed point theorem in Menger spaces. Int. Math. J., 1: 1543-1554.

7. Milovanovic-Arandelovic, M.M., 1997. A common fixed points theorem for contraction type mappings on Menger spaces..Filomat, 11: 103-108.

8. Radu, V., 1994. Lectures on probabilistic analysis, Timişoara University, Surveys Lectures Notes and Monograph Series on Probability Statistics and Applied Mathematics. Vol. 2.

9. Singh, B. and S. Jain, 2005. A fixed point theorem in Menger Space through weak compatibility. J. Math. Anal. Appl., 301: 439-448.

10. Vasuki, R., 1990. A fixed point theorem for a sequence of maps satisfying a new contraction type condition in Menger spaces. Math. Japonica, 35: 1099-1102.

11. Constantin, G. and I. Istratescu, 1989. Elements of probabilistic analysis, Ed. Acad. Bucureşti and Kluwer Acad. Publ.

12. Kutukcu, S., D. Turkoglu and C. Yildiz, 2006. Common fixed points of compatible maps of type $(\beta)$ on fuzzy metric spaces. Commun. Korean Math. Soc., 21: 89-100.

13. Schweizer, B., A. Sklar and E. Thorp, 1960. The metrization of SM-spaces. Pasific J. Math., 10: 673-675. 